\begin{tabular}{|c|c|c|}
\hline Title: & \multicolumn{2}{|c|}{$\begin{array}{l}\text { A Novel } 100 \text { kW Power Hardware-in-the-Loop Emulation Test Bench for Permanent } \\
\text { Magnet Synchronous Machines with Nonlinear Magnetics }\end{array}$} \\
\hline Authors: & \multicolumn{2}{|c|}{ Alexander Schmitt, Jan Richter, Mario Gommeringer, Thomas Wersal, Michael Braun } \\
\hline Institute: & \multicolumn{2}{|c|}{$\begin{array}{l}\text { Karlsruhe Institute of Technology (KIT) } \\
\text { Elektrotechnisches Institut (ETI) }\end{array}$} \\
\hline Type: & \multicolumn{2}{|c|}{ Conference Proceedings } \\
\hline Published at: & $\begin{array}{l}\text { The } 8^{\text {th }} \text { IET Internation } \\
\text { (PEMD) } 2016 \\
\text { Date of Conference: } \\
\text { Conference Location: } \\
\text { Publisher: } \\
\text { ISBN: }\end{array}$ & $\begin{array}{l}\text { Conference on Power Electronics, Machines and Drives } \\
\text { 19. April } 2016-21 \text {. April } 2016 \\
\text { Glasgow, Scotland, UK } \\
\text { The Institution of Engineering and Technology (IET) } \\
978-1-78561-189-6\end{array}$ \\
\hline
\end{tabular}




\title{
A Novel 100 kW Power Hardware-in-the-Loop Emulation Test Bench for Permanent Magnet Synchronous Machines with Nonlinear Magnetics
}

\author{
Alexander Schmitt, Jan Richter, Mario Gommeringer, Thomas Wersal, Michael Braun \\ Karlsruhe Institute of Technology (KIT) - Elektrotechnisches Institut (ETI) \\ Kaiserstr. 12, 76131 Karlsruhe, Germany \\ a.schmitt@kit.edu
}

Keywords: Power Hardware-in-the-Loop, DC-AC power converters, System testing, Virtual machine

\begin{abstract}
This paper presents a high dynamic power hardware-inthe-loop (PHIL) emulation test bench to mimic arbitrary permanent magnet synchronous machines with nonlinear magnetics. The proposed PHIL test bench is composed of a high performance real-time simulation system to calculate the machine behaviour and a seven level modular multiphase multilevel converter to emulate the power flow of the virtual machine. The PHIL test bench is parametrized for an automotive synchronous machine and controlled by a motor converter using a predictive trajectory dead-beat current controller. Measurements of high dynamic current steps and phase current ripples at the real machine are reproduced precisely at the PHIL test bench. Thus, the validity of the used machine model as well as the excellent performance of the PHIL test bench is proven.
\end{abstract}

\section{Introduction}

In modern motor converter development processes the need for advanced test benches increases rapidly. Thus, besides various state of the art computer simulation tools, real-time hardware-in-the-loop (HIL) test benches were developed [1, $2,3,4]$. However, HIL test benches are not suitable to test the manufacturing processes and the reliability or the behaviour of the entire device under various fault conditions [5]. Hence, conventional motor load test benches (Figure 1 (b)) are used for that purpose. Unfortunately, there are several drawbacks and disadvantages inherent to conventional motor load testbeds. Due to the fact that there are numerous different applications that drives can be used for, various motor load combinations are required to test the drive system. Furthermore, as a result of fabrication tolerances, the test conditions can vary between actually identical motor load combinations. Besides, conventional test benches are strongly limited in their fault emulation capability. Especially fault conditions like a blocking rotor or a crack of the shaft are very difficult to test. Therefore, it is desirable to connect the converter to a free configurable power hardware-in-the-loop (PHIL) emulation test bench for arbitrary motor load combinations.

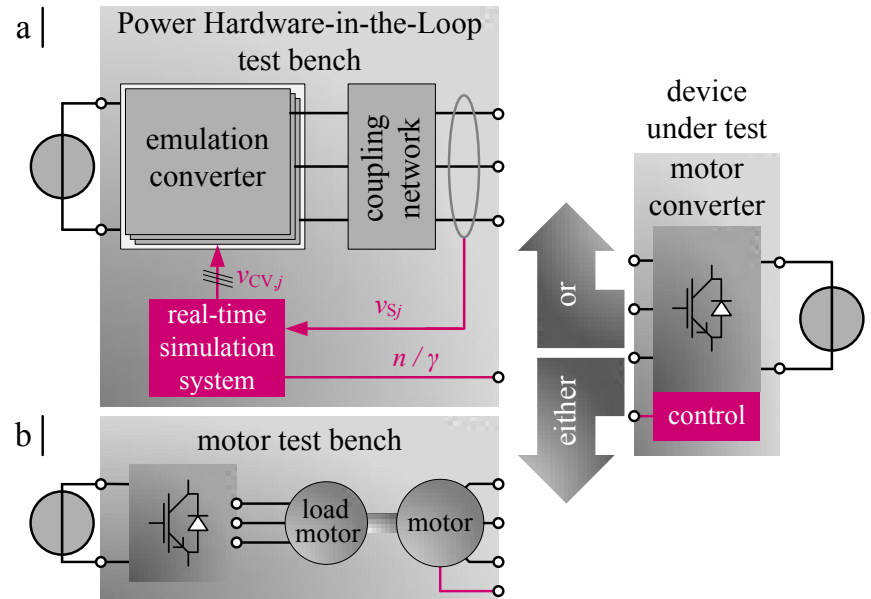

Figure 1: Schematic diagram of a motor converter either connected to a PHIL test bench (a) or a conventional motor test bench (b).

Figure 1 (a) shows a typical PHIL emulation test bench. At this type of test bench, the HIL real-time simulation system is extended by an emulation converter which is connected to the device under test (DUT) through an inductive coupling network. Since the DUT commonly controls the machine currents, the PHIL emulation system should control the counter voltages $v_{\mathrm{CV}, j}$ at the coupling network to avoid problems caused by a second current controller within the PHIL [6]. Therefore, the coupling network has to be fed by a high-precision $3 \mathrm{AC}$ voltage source converter to ensure the correct current slopes of the real machine within the PHIL test bench [7]. Thereby, the counter voltages have to be calculated by the real-time simulation system taking the voltage drop of the coupling network into account [8].

In this paper, a novel PHIL test bench for the emulation of arbitrary permanent magnet synchronous machines (PMSM) with nonlinear magnetics is introduced. The theory of the underlying machine model is briefly described in Section 2. Subsequently, the emulation concept and the used control scheme of the PHIL test bench is presented in Section 3. In addition, measurement results of the PHIL test bench and of a real motor using a predictive trajectory controller are presented in Section 4. Conclusions are stated in Section 5.

\section{Theory}

The intention of the proposed power hardware-in-the-loop 


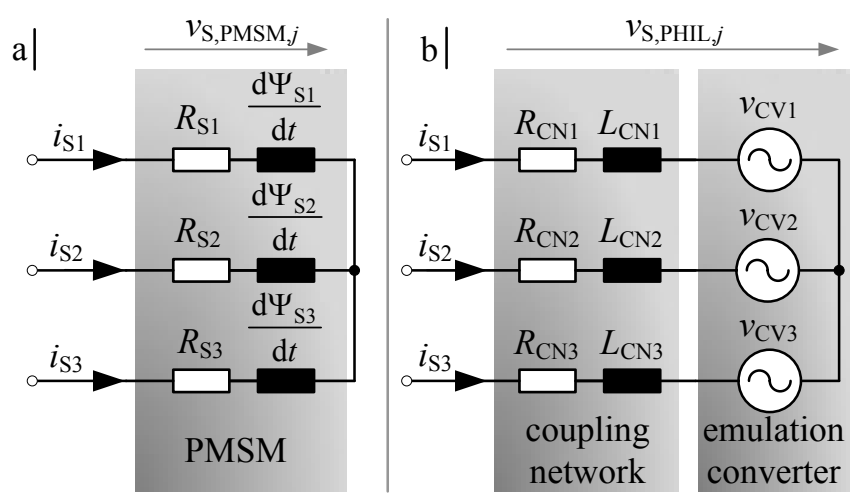

Figure 2: Equivalent circuit of a PMSM (a) and equivalent circuit of the power hardware-in-the-loop test bench (b).

test bench is the emulation of the terminal behaviour of arbitrary permanent magnet synchronous machines (PMSM). The magnetic anisotropy as well as iron saturation, including cross-saturation have to be considered in the machine model because PMSM often have a reluctance torque and are highly utilized, especially in automotive applications. Figure 2 (a) depicts the general equivalent circuit of such a PMSM. In this circuit, with $j \in\{1,2,3\}$ as phase number, the voltage equation of a single PMSM phase is:

$$
\mathrm{v}_{\mathrm{S} j}=R_{\mathrm{S} j} \cdot i_{\mathrm{S} j}+\frac{\mathrm{d} \Psi_{\mathrm{S} j}}{\mathrm{~d} t}
$$

Therein, the stator flux linkages $\Psi_{\mathrm{S} j}=\Psi_{\mathrm{S} j}\left(i_{\mathrm{S} 1}, i_{\mathrm{S} 2}, i_{\mathrm{S} 3} \gamma\right)$ depend on the three stator currents $i_{\mathrm{S} j}$ and the electric rotor position angle $\gamma$ [8]. In comparison, Figure 2 (b) illustrates the equivalent circuit of the proposed PHIL test bench. The phase voltages $v_{\mathrm{S}, \mathrm{PHIL}, j}$ of the PHIL test bench must be identical to the phase voltages $v_{\mathrm{S} j}$ of the PMSM to emulate the physical behaviour of the PMSM. For this reason, the counter voltages $v_{\mathrm{CV} j}$ have to be calculated, taking the voltage drop at the coupling network into account. This is obtained by subtracting the voltage drop from the derivation of the flux linkages $\Psi_{\mathrm{S} j}$ and leads to [8]:

$$
\begin{aligned}
v_{\mathrm{S} j} & =v_{\mathrm{S}, \mathrm{PHIL}, j}=R_{\mathrm{CN} j} \cdot i_{\mathrm{S} j}+L_{\mathrm{CN} j} \cdot \frac{\mathrm{d} i_{\mathrm{S} j}}{\mathrm{~d} t} \\
+ & \underbrace{\left(\frac{\mathrm{d} \Psi_{\mathrm{S} j}}{\mathrm{~d} t}-L_{\mathrm{CN} j} \cdot \frac{\mathrm{d} i_{\mathrm{S} j}}{\mathrm{~d} t}-\left(R_{\mathrm{CN} j}-R_{\mathrm{S} j}\right) \cdot i_{S j}\right)}_{v_{\mathrm{CV} j}}
\end{aligned}
$$

Assuming that the three inductors $L_{\mathrm{CN} j}$ as well as the three ohmic resistors $R_{\mathrm{CN} j}$ are linear and identical, the phase elements $i_{\mathrm{S} j}, v_{\mathrm{S} j}$ and $\Psi_{\mathrm{S} j}$ can be transformed to the direct (d) and quadrature (q) reference frame with $\underline{a}=e^{j \frac{2 \pi}{3}}$ and $\underline{z} \in\left\{\Psi_{\mathrm{S}}, v_{\mathrm{S}}, i_{\mathrm{S}}\right\}$ by:

$$
\underline{z}=\frac{2}{3} \cdot\left(x_{1}+\underline{a} \cdot x_{2}+\underline{a}^{2} \cdot x_{3}\right) \cdot e^{-\mathrm{j} \gamma}
$$

This yields:
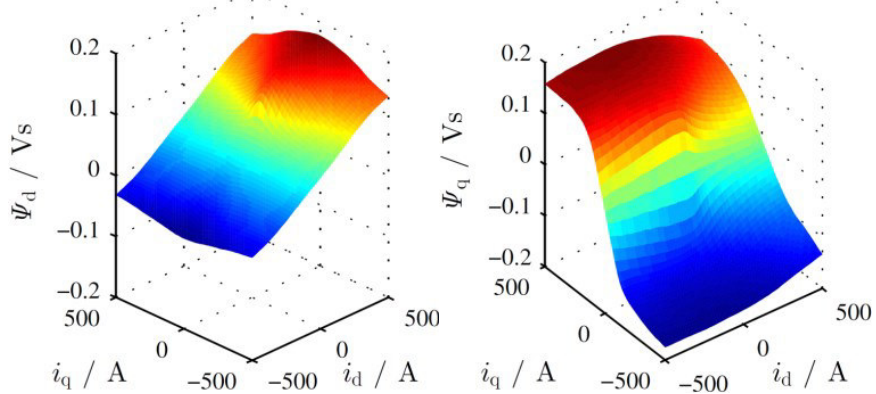

Figure 3: Flux linkage $\Psi_{\mathrm{d}}$ (left) and flux linkage $\Psi_{\mathrm{q}}$ (right) [8].

$$
\begin{aligned}
v_{\mathrm{d}}= & R_{\mathrm{CN}} \cdot i_{\mathrm{d}}+L_{\mathrm{CN}} \frac{\mathrm{d} i_{\mathrm{d}}}{\mathrm{d} t}+\left(-\omega \cdot L_{\mathrm{CN}} \cdot i_{\mathrm{q}}+\frac{\mathrm{d} \Psi_{\mathrm{d}}}{\mathrm{d} t}-\omega \Psi_{\mathrm{q}}\right. \\
& \left.-L_{\mathrm{CN}} \cdot \frac{\mathrm{d} i_{\mathrm{d}}}{\mathrm{d} t}+\omega \cdot L_{\mathrm{CN}} \cdot i_{\mathrm{q}}-\left(R_{\mathrm{CN}}-R_{\mathrm{S}}\right) \cdot i_{\mathrm{d}}\right) \\
v_{\mathrm{q}}= & R_{\mathrm{CN}} \cdot i_{\mathrm{q}}+L_{\mathrm{CN}} \frac{\mathrm{d} i_{\mathrm{q}}}{\mathrm{d} t}+\left(\omega \cdot L_{\mathrm{CN}} \cdot i_{\mathrm{d}}+\frac{\mathrm{d} \Psi_{\mathrm{q}}}{\mathrm{d} t}+\omega \Psi_{\mathrm{d}}\right. \\
& \left.-L_{\mathrm{CN}} \cdot \frac{\mathrm{d} i_{\mathrm{q}}}{\mathrm{d} t}-\omega \cdot L_{\mathrm{CN}} \cdot i_{\mathrm{d}}-\left(R_{\mathrm{CN}}-R_{\mathrm{S}}\right) \cdot i_{\mathrm{q}}\right)
\end{aligned}
$$

In this representation, the flux linkages $\Psi_{\mathrm{d}}$ and $\Psi_{\mathrm{q}}$ depend nonlinearly on the currents $i_{\mathrm{d}}$ and $i_{\mathrm{q}}$ due to iron saturation as defined by the function $f$ and shown in Figure 3 .

$$
f: \mathbb{R}^{2} \rightarrow \mathbb{R}^{2}, \quad\left(i_{\mathrm{d}}, i_{\mathrm{q}}\right) \mapsto\left(\Psi_{\mathrm{d}}, \Psi_{\mathrm{q}}\right)
$$

The flux linkages can be obtained by finite-element method calculations [9] or by stationary measurements of the machine [10]. The machine model is used in a real-time simulator as illustrated in Figure 1 (a) to calculate the counter voltage of the emulation converter [8]. Thus, the output voltages $v_{\mathrm{S} j}$ of the device under test are measured. Subsequently, using (4) and (5), the derivatives of the machine currents can be calculated to [8]:

$$
\begin{aligned}
& \frac{\mathrm{d} i_{\mathrm{d}}}{\mathrm{d} t}=\frac{v_{\mathrm{d}}-R_{\mathrm{S}} i_{\mathrm{d}}+\frac{L_{\mathrm{dq}}}{L_{\mathrm{qq}}}\left(-v_{\mathrm{q}}+R_{\mathrm{S}} i_{\mathrm{q}}+\omega \Psi_{\mathrm{d}}\right)+\omega \Psi_{\mathrm{q}}}{L_{\mathrm{dd}}-\frac{L_{\mathrm{dq}} \cdot L_{\mathrm{qd}}}{L_{\mathrm{qq}}}} \\
& \frac{\mathrm{d} i_{\mathrm{q}}}{\mathrm{d} t}=\frac{v_{\mathrm{q}}-R_{\mathrm{S}} i_{\mathrm{q}}+\frac{L_{\mathrm{qd}}}{L_{\mathrm{dd}}}\left(-v_{\mathrm{d}}+R_{\mathrm{S}} i_{\mathrm{d}}-\omega \Psi_{\mathrm{q}}\right)-\omega \Psi_{\mathrm{d}}}{L_{\mathrm{qq}}-\frac{L_{\mathrm{dq}} \cdot L_{\mathrm{qd}}}{L_{\mathrm{dd}}}}
\end{aligned}
$$

Therein, $L_{x y}$ with $(x, y \in\{\mathrm{d}, \mathrm{q}\})$ are differential inductances and thus partial derivatives of the flux linkage function in the direct and quadrature direction. Furthermore, the counter voltage $v_{\mathrm{CV}, \mathrm{d}}$ and $v_{\mathrm{CV}, \mathrm{q}}$ considering the voltage drop at the coupling network can be calculated by:

$$
\begin{aligned}
v_{\mathrm{CV}, \mathrm{d}}= & i_{\mathrm{q}} \cdot \omega \cdot L_{\mathrm{CN}}+\frac{\mathrm{d} i_{\mathrm{d}}}{\mathrm{d} t}\left(L_{\mathrm{dd}}-L_{\mathrm{CN}}\right)+\frac{\mathrm{d} i_{\mathrm{q}}}{\mathrm{d} t} L_{\mathrm{dq}} \\
& -\omega \Psi_{\mathrm{q}}-\left(R_{\mathrm{CN}}-R_{\mathrm{S}}\right) \cdot i_{\mathrm{d}}
\end{aligned}
$$




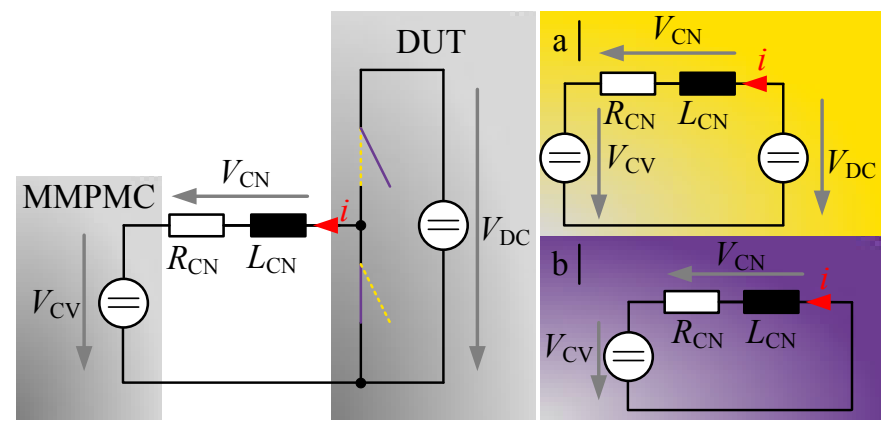

Figure 4: Illustration of the equivalent circuit of a single phase PHIL test bench for an active switching state (a) and a freewheeling state (b) of the DUT.

$$
\begin{aligned}
v_{\mathrm{CV}, \mathrm{q}}= & -i_{\mathrm{d}} \cdot \omega \cdot L_{\mathrm{CN}}+\frac{\mathrm{d} i_{\mathrm{q}}}{\mathrm{d} t}\left(L_{\mathrm{qq}}-L_{\mathrm{CN}}\right)+\frac{\mathrm{d} i_{\mathrm{d}}}{\mathrm{d} t} L_{\mathrm{qd}} \\
& +\omega \Psi_{\mathrm{d}}-\left(R_{\mathrm{CN}}-R_{\mathrm{S}}\right) \cdot i_{\mathrm{q}}
\end{aligned}
$$

Finally, the machine model is completed by the equations for the inner torque $T_{\mathrm{i}}$ :

$$
T_{\mathrm{i}}=\frac{3}{2} \cdot p\left(\Psi_{\mathrm{d}} \cdot i_{\mathrm{q}}-\Psi_{\mathrm{q}} \cdot i_{\mathrm{d}}\right)
$$

and the equation for the electric rotor speed $\omega$ :

$$
\omega=\frac{p}{J} \int\left(T_{\mathrm{i}}-T_{\mathrm{L}}\right) \mathrm{d} t
$$

whereby, $J$ is the inertia torque, $T_{\mathrm{L}}$ equals the load torque and $p$ is the number of pole pairs of the machine.

\section{Emulation Concept}

Due to the performance of modern FPGAs and A/Dconverters it is possible to calculate the derived machine equations with sample rates of more than $1 \mathrm{MHz}$ quasi continuous in real-time. Indeed, the real-time emulation of the power flow is more challenging, especially for high power applications. Thereby, the difficulty is the emulation of variable inductances e.g. of PMSM due to iron saturation or the magnetic anisotropy of the rotor. To overcome this, the counter voltage at the coupling network has to be changed very dynamically dependent on the switching state of the DUT. Figure 4 illustrates the simplified equivalent circuit of a single phase PHIL test bench for an active switching state (a) and a freewheeling state (b) of the DUT. Neglecting the ohmic resistance, the counter voltage $v_{\mathrm{CV}}$ that ensures the desired current slopes $\frac{\mathrm{d} i_{\mathrm{W}}}{\mathrm{d} t}$ within the constant inductance $L_{\mathrm{CN}}$ for an active switching state is:

$$
v_{\mathrm{CV}}=V_{\mathrm{DC}}-L_{\mathrm{CN}} \cdot \frac{\mathrm{d} i_{\mathrm{w}}}{\mathrm{d} t}
$$

And for a freewheeling state of the DUT:

$$
v_{\mathrm{CV}}=-L_{\mathrm{CN}} \cdot \frac{\mathrm{d} i_{\mathrm{w}}}{d t}
$$

For this reason, modelling of the current slopes $\frac{\mathrm{d} i_{\mathrm{w}}}{\mathrm{d} t}$ requires a converter topology that allows a high dynamic and very precise generation of the counter voltages $v_{\mathrm{CV} j}$. Therefore, the modular multiphase multilevel converter (MMPMC) [11], in conjunction with the modulation schemes from [7] is used as

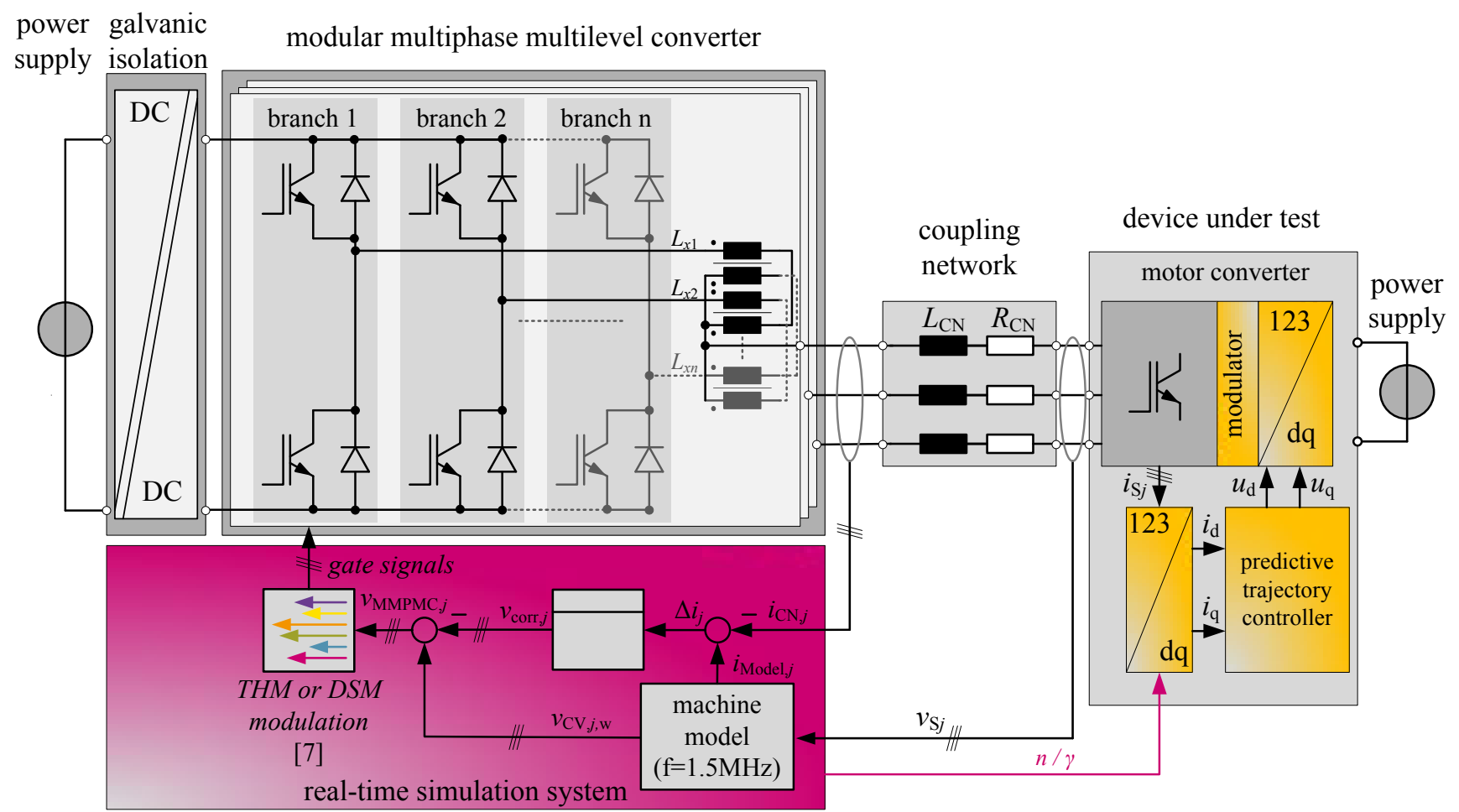

Figure 5: Detailed schematic diagram of the proposed PHIL test bench including the modular multiphase multilevel converter and the realtime simulation system as well as the coupling network and the device under test. 
Table 1

Machine Parameters

\begin{tabular}{l|c}
\hline Parameter & Value \\
\hline line voltage nom. & $212 \mathrm{~V}$ \\
\hline current nom. /max. & $169 \mathrm{~A} / 300 \mathrm{~A}$ \\
\hline shaft power nom. / max. & $57 \mathrm{~kW} / 97 \mathrm{~kW}$ \\
\hline speed nom. / max. & $4200 \mathrm{~min}^{-1} /$ \\
& $11000 \mathrm{~min}^{-1}$ \\
\hline torque nom. / max. & $130 \mathrm{Nm} / 220 \mathrm{Nm}$ \\
\hline inertia torque & $0.06 \mathrm{~kg} \cdot \mathrm{m}^{2}$ \\
\hline ohmic stator resistance typ. & $10.5 \mathrm{~m} \Omega$ \\
\hline number of pole pairs & 3 \\
\hline$L_{\mathrm{dd}}(0 A, 0 A) / L_{\mathrm{dd}}(-200 A, 200 A)$ & $410 \mu \mathrm{H} / 204.5 \mu \mathrm{H}$ \\
\hline$L_{\mathrm{qq}}(0 A, 0 A) / L_{\mathrm{qq}}(-200 A, 200 A)$ & $2.1 \mathrm{mH} / 163.6 \mu \mathrm{H}$ \\
\hline \multicolumn{2}{c}{ Coupling Network }
\end{tabular}

\begin{tabular}{l|c}
\hline $\begin{array}{l}\text { ohmic resistance of the coupling } \\
\text { network typ. }\end{array}$ & $17.5 \mathrm{~m} \Omega$ \\
\hline inductance of the coupling network & $1 \mathrm{mH}$ \\
\hline
\end{tabular}

emulation converter to generate the required counter voltages. The schematic diagram of the entire PHIL test bench is shown in Figure 5. The proposed test bench is composed of a MMPMC with $n=6$ branches per phase. Hence, it generates a seven level output voltage waveform with a resulting PWM frequency of $f_{\mathrm{PWM}}=120 \mathrm{kHz}$ [7]. The MMPMC is fed by a six phase resonant push pull converter to ensure the galvanic isolation of the test bench identically to the real machine coils. Since the MMPMC has the behaviour of a voltage source, an external coupling network is necessary to connect the DUT inverter to the PHIL test bench.

The entire PHIL test bench is controlled by an FPGA-based real-time simulation system. The real-time simulation system contains the machine model, derived in Section 2, as well as the modulation of the MMPMC [7]. Additionally, the realtime simulation system contains a P-controller. This Pcontroller is necessary to avoid a drift of the inner model currents $i_{\text {Model }, j}$ and the real currents $i_{\mathrm{CN}, j}$ in the coupling network due to the open integration of the machine currents in the FPGA. A difference between these currents would affect the calculation of the counter voltages and the inner torque. Hence, it would distort the physical behaviour of the PHIL test bench compared to the real machine. Indeed, due to the very precise realization of the counter voltage, a simple Pcontroller is sufficient and furthermore does not affect the stability of the current controller of the DUT. In addition, the real-time simulation system is able to emulate an incremental encoder as well as a resolver. Identically to real machines, this sensor signal is the only connection between PHIL and DUT besides the three power terminals.

\section{Measurements}

Figure 6 (c) shows the DUT to validate the concept and the performance of the proposed PHIL test bench. This motor converter is based on a Semikron SkiiP (513GD122-3DUL) a) PHIL test bench

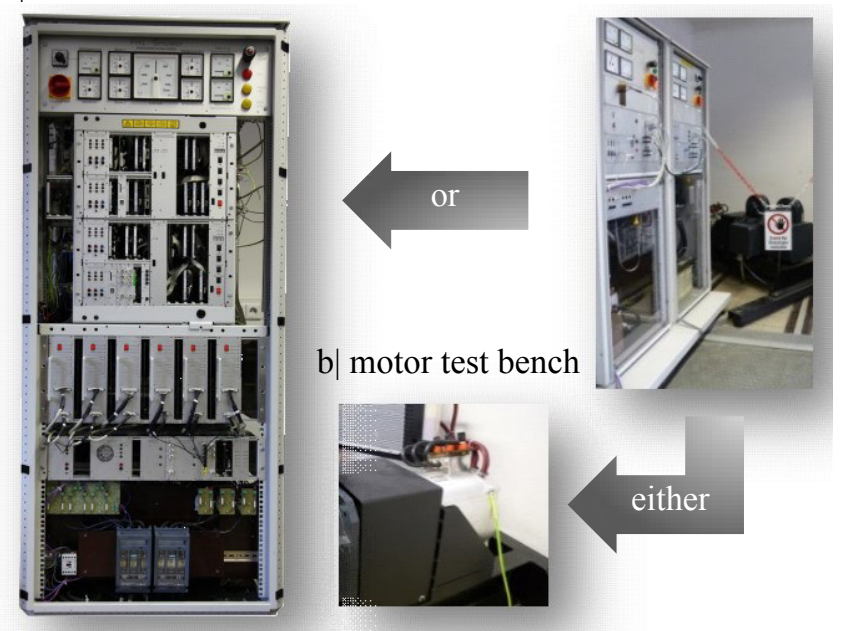

Figure 6: Test bench components including the PHIL test bench (a), the real PMSM (b) and the DUT motor converter (c)

six-pulse bridge and is controlled by the predictive trajectory controller which was proposed in [10]. During the measurements, the integral component of the controller was disabled so that model errors of the PHIL test bench are not compensated by the controller. During the measurements, the DUT is first connected to the PHIL test bench (Figure 6 (a)) and afterwards to a real motor test bench (Figure 6 (b)). Subsequently, the measurement results are compared. Thereby, the test bench machine is a motor manufactured by Brusa of type HSM1-6.1712-C01. This machine is an interior permanent magnet synchronous machine with a strong reluctance torque. Figure 3 illustrates the measured d- and qflux linkages. These flux linkages are used for both the predictive trajectory controller and the machine model of the PHIL test bench. Table 1 contains the basic parameters of the machine, as well as the parameters of the coupling network. It has to be considered that due to the interior permanent magnets, the differential inductances of the machine $L_{\mathrm{dd}}$ and $L_{\mathrm{qq}}$ differ by a factor of approximately five at a current of $i_{\mathrm{d}, \mathrm{q}}=0 \mathrm{~A} \quad\left(L_{\mathrm{qq}, 0 \mathrm{~A}} \approx 5 \cdot L_{\mathrm{dd}, 0 \mathrm{~A}} \approx 2 \mathrm{mH}\right)$. In contrast, the inductances at a current of $i_{\mathrm{d}}=-200 \mathrm{~A}$ and $i_{\mathrm{q}}=200 \mathrm{~A}$ are strongly decreased to some hundreds of micro henry and are approximately equal $\left(L_{\mathrm{qq}, 200 \mathrm{~A}} \approx L_{\mathrm{dd}, 200 \mathrm{~A}} \approx 200 \mu \mathrm{H}\right)$. However, the inductors of the coupling network have a constant inductance of $1 \mathrm{mH}$ which is basically the middle of the possible machine inductances. The measurements are carried out at a DC-link voltage of $300 \mathrm{~V}$ at the DUT and a DC-link voltage of $650 \mathrm{~V}$ at the MMPMC. The real machine was set to a constant rotational speed of $1000 \mathrm{~min}^{-1}$ by means of a speed controlled load motor which is connected to the PMSM. The rotational speed of the virtual machine was set to $1000 \mathrm{~min}^{-1}$ by software. Furthermore, the current controller of the DUT is executed with a control frequency of $f_{\mathrm{c}}=8 \mathrm{kHz}$ why the switching and sampling frequency of the DUT is also $8 \mathrm{kHz}$. The machine model of the emulator is calculated with a sampling rate of $1.5 \mathrm{Mhz}$. In addition, the threshold modulation [7] is used to generate the counter voltages $v_{\mathrm{CV} j}$ with a PWM frequency of $f_{\mathrm{PWM}}=120 \mathrm{kHz}$. For this 
a| Trend of the output currents at the PHIL test bench

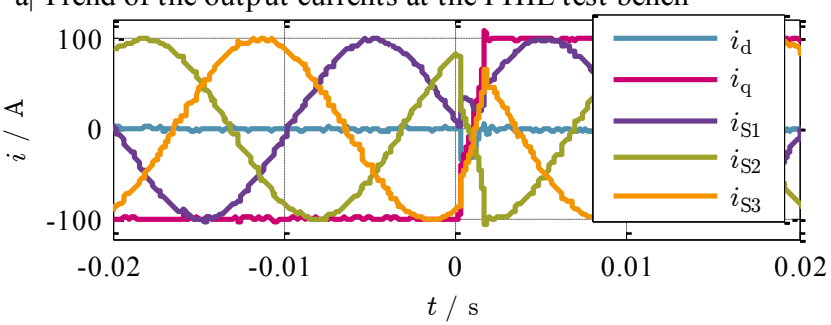

b| Trend of the output currents at the real PMSM

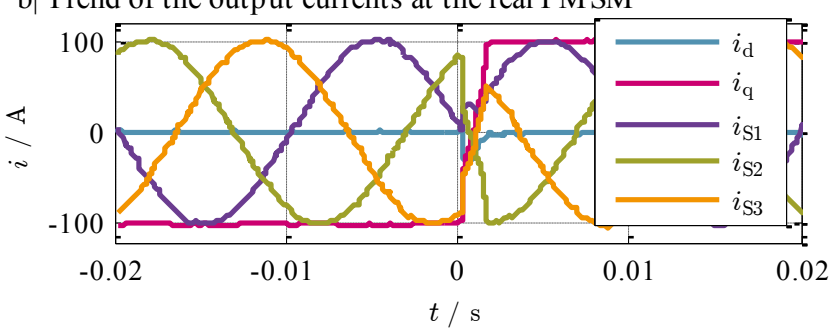

c| Comparison of the d- and q- currents

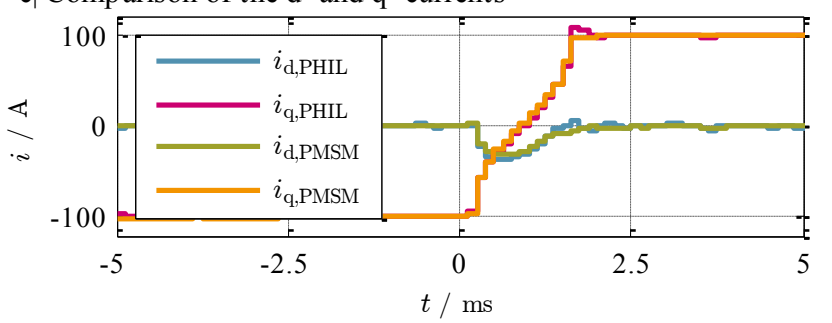

d| Difference of the currents at PMSM and PHIL

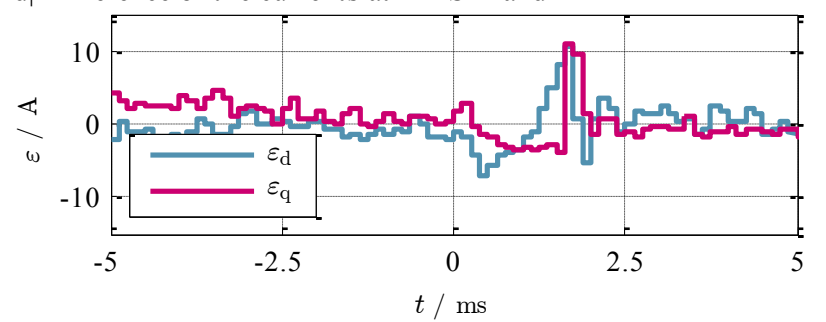

Figure 7: Trend of the sampled phase currents for a q-current step from $i_{\mathrm{q}, \mathrm{w}}=-100 \mathrm{~A}$ to $i_{\mathrm{q}, \mathrm{w}}=100 \mathrm{~A}$ at the PHIL test bench (a) and at the real PMSM (b). Additionally, a comparison of the d- and q- currents (c) and the calculated difference between the PHIL test bench and the real machine is shown (d).

reason, the set values of the counter voltages are previously averaged over one modulation period $T_{\mathrm{PWM}}=\frac{1}{f_{\mathrm{PWM}}}$.

Figure 7 (a) depicts the trend of the phase currents of the DUT during a current step at the PHIL test bench. Thereby, the set value is changed form $i_{\mathrm{q}, \mathrm{w}}=-100 \mathrm{~A}$ to $i_{\mathrm{q}, \mathrm{w}}=100 \mathrm{~A}$ at an electric rotor angle of $\gamma=\pi$. Figure 7 (b) shows the absolute identical current step of the DUT connected to the real PMSM. A detailed comparison of the currents during the current step is shown in Figure 7 (c) to illustrate the accuracy of the test bench. It can be seen that not only the currents in the q-axis but also the currents in the d-axis, which change due to cross-saturation, are nearly identical even for a high dynamic current step at the physical limits of the machine. Furthermore, it can be seen that the q-current increases nonlinearly due to saturation. Figure 7 (d) shows the difference $\varepsilon_{\mathrm{d}, \mathrm{q}}=i_{\mathrm{d}, \mathrm{q}, \mathrm{PHIL}}-i_{\mathrm{d}, \mathrm{q}, \mathrm{PMSM}}$ of the sampled currents a| Comparison of the d- and q- currents

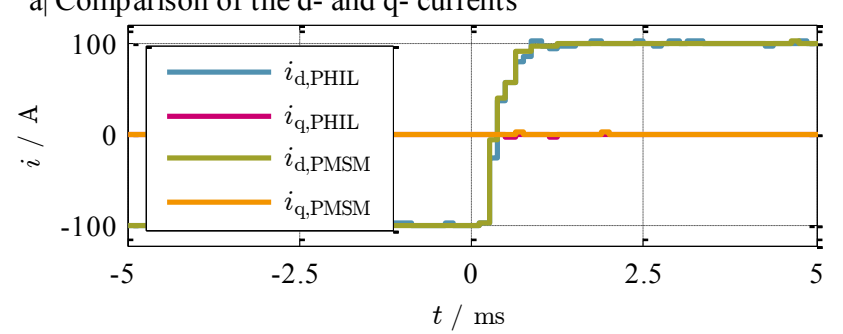

b) Difference of the currents at PMSM and PHIL

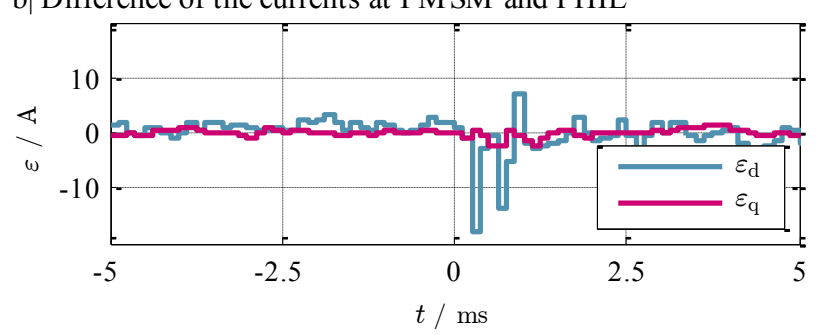

Figure 8: Comparison of the sampled d- and q-currents during a dcurrent step from $i_{\mathrm{d}, \mathrm{w}}=-100 \mathrm{~A}$ to $i_{\mathrm{d}, \mathrm{w}}=100 \mathrm{~A}$ at the PHIL test bench and at the real PMSM (a). The calculated difference between the PHIL test bench and the real machine is shown in (b).

during the step. It can be seen that the differences $\varepsilon_{\mathrm{d}, \mathrm{q}}$ increase during the step and are $\varepsilon_{\mathrm{d}, \mathrm{q}}<10 \mathrm{~A}$, respectively $\varepsilon_{\mathrm{d}, \mathrm{q}}<5 \%$ of $\Delta i_{\mathrm{q}, \mathrm{w}}$. The causes of this difference are inaccuracies of the measurement at the DUT and the real-time simulation system, integration inaccuracies due to the discrete calculation of the machine model as well as the dead time of the counter voltage generation of the MMPMC. Indeed, these small differences do not affect the performance, stability or accuracy of the used current controller since the physical behaviour of the emulated machine is despite these inaccuracies identical to the real machine.

Moreover, Figure 8 (a) depicts a step in the d-axis also from $i_{\mathrm{d}, \mathrm{w}}=-100 \mathrm{~A}$ to $i_{\mathrm{d}, \mathrm{w}}=100 \mathrm{~A}$. As aforementioned, the inductance in the $\mathrm{d}$-axis is significant smaller than the inductance in the q-axis. Nevertheless, besides the excellent accuracy of the PHIL emulation in the d-axis, Figure 8 (a) illustrates also the significant higher dynamic of the current control in the d-axis compared to the q-axis. Furthermore, this plot depicts that the test bench is capable to emulate arbitrary inductances over a wide range. The difference of the currents between the real machine and the PHIL test bench during a dcurrent step is $\varepsilon_{\mathrm{d}}<18 \mathrm{~A}$ whereas the difference in the qcurrent is $\varepsilon_{\mathrm{q}}<5 \mathrm{~A}$ (Figure 8 (b)). In addition, it can be seen that the emulation of the machine is possible in the entire current plane. Indeed, the operation at positive d-currents is not reasonable in a practical application.

Furthermore, Figure 9 shows a comparison of a torque step from $M_{\mathrm{i}}=0 \mathrm{Nm}$ to $M_{\mathrm{i}}=100 \mathrm{Nm}$ at the maximum torque per current (MTPC) line of the machine why the d-and q-currents are changed simultaneously. Again, the differences of the currents $\varepsilon_{\mathrm{d}, \mathrm{q}}$ are smaller than $\varepsilon_{\mathrm{d}}<20 \mathrm{~A}$ in the d-axis and $\varepsilon_{\mathrm{q}}<10 \mathrm{~A}$ in the q-axis.

Finally, the time response of the phase currents is evaluated. Figure 10 shows a detailed oscillogram of the phase current $i_{\mathrm{S} 1}$ at the PHIL test bench and at the real PMSM for a set value of $i_{\mathrm{q}, \mathrm{w}}=100 \mathrm{~A}$. The phase currents are measured using 


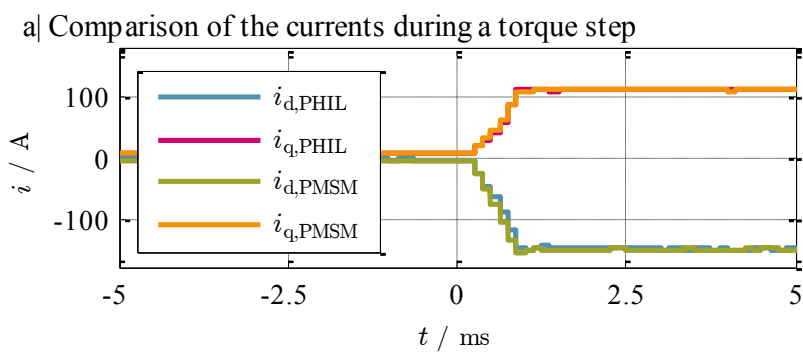

b| Difference of the currents at PMSM and PHIL

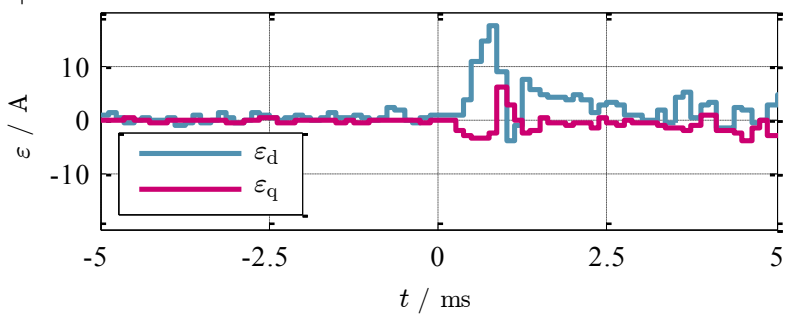

Figure 9: Torque step from $M_{\mathrm{i}}=0 \mathrm{Nm}$ to $M_{\mathrm{i}}=100 \mathrm{Nm}$ at the PHIL test bench and the real PMSM (a) and calculated difference of the sampled currents between the PHIL test bench and the real machine (b)

the complete identical measurement setup (probes, settings and scales) and plotted without further adaption. As it can be seen, even the current ripples at the PMSM (orange) and the current ripple at the PHIL test bench (purple) are very similar. As expected, the counter voltage $v_{\mathrm{CV} 1}$ (green) has to change very dynamically during the space vector modulation period $T_{\mathrm{c}}$ of the DUT to ensure the correct current slopes within the inductors of the coupling network. Furthermore, it has to be considered that the current ripple characteristics are identical in the middle of the current slopes. This is the time where the current controller commonly samples the phase currents.

\section{Conclusion}

This paper has presented a novel power hardware-in-the-loop (PHIL) emulation test bench for arbitrary permanent magnet synchronous machines. The underlying machine model considers iron saturation as well as the magnetic anisotropy of the rotor. Furthermore, the PHIL concept using a $100 \mathrm{~kW}$ seven level modular multiphase multilevel converter is introduced and verified. Therefore, the PHIL test bench is parametrized for an automotive PMSM and controlled by a DUT using a predictive trajectory dead-beat current controller. Measurements of high dynamic current steps and the phase current ripples at the real machine are reproduced precisely at the PHIL test bench. Thus, the validity of the used machine model as well as the excellent performance of the PHIL test bench is proven.

\section{References}

[1] C. Dufour, S. Cense, T. Yamada, R. Imamura, and J. Belanger, "Fpga permanent magnet synchronous motor floating-point models with variable-dq and spatial harmonic finite-element analysis solvers," in 15th International Power Electronics and Motion Control Conference (EPE/PEMC), 2012, pp. LS6b.2-1LS6b.2-10.

[2] M. Matar and R. Iravani, "Massively parallel implementation of

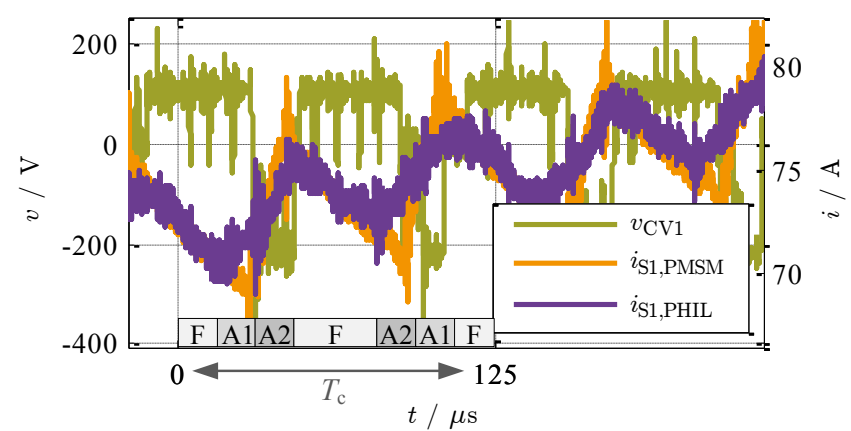

Figure 10: Oscillogram of the current ripple $i_{\mathrm{S} 1, \mathrm{PMSM}}$ of the real PMSM (orange) and the current ripple $i_{\mathrm{S} 1, \mathrm{PHIL}}$ at the PHIL test bench (purple) as well as the corresponding counter voltage $v_{\mathrm{CV} 1}$ (green) which is applied to the coupling network.

ac machine models for fpga-based real-time simulation of electromagnetic transients," IEEE Transactions on Power Delivery, vol. 26, no. 2, pp. 830-840, 42011.

[3] L. Herrera and J. Wang, "Fpga based detailed real-time simulation of power converters and electric machines for ev hil applications," in IEEE Energy Conversion Congress and Exposition (ECCE), 9 2013, pp. 1759-1764.

[4] M. Oettmeier and C. Heising, "Fpga-based pmsm emulation: Concept and verification," International Exhibition and Conference for Power Electronics, Intelligent Motion, Renewable Energy and Energy Management; Proceedings of PCIM Europe, 2012.

[5] A. Bouscayrol, "Different types of hardware-in-the-loop simulation for electric drives," in IEEE International Symposium on Industrial Electronics ISIE, 2008, pp. 2146-2151.

[6] R. Kennel, T. Boller, and J. Holtz, "Replacement of electrical (load) drives by a hardware-in-the-loop system," in International Aegean Conference on Electrical Machines and Power Electronics (ACEMP) and Electromotion Joint Conference, 2011, pp. 17-25.

[7] A. Schmitt, M. Gommeringer, C. Rollbühler, P. Pomnitz, and M. Braun, "A novel modulation scheme for a modular multiphase multilevel converter in a power hardware-in-the-loop emulation system," in IECON 2015 - 41th Annual Conference of the IEEE Industrial Electronics Society, Nov 2015, pp. 12761281.

[8] A. Schmitt, J. Richter, U. Jurkewitz, and M. Braun, "FPGAbased real-time simulation of nonlinear permanent magnet synchronous machines for power hardware-in-the-loop emulation systems," in IECON 2014 - 40th Annual Conference of the IEEE Industrial Electronics Society, Oct 2014, pp. 3763 3769.

[9] X. Chen, J. Wang, B. Sen, P. Lazari, and T. Sun, "A highfidelity and computationally efficient model for interior permanent-magnet machines considering the magnetic saturation, spatial harmonics, and iron loss effect," IEEE Transactions on Industrial Electronics, vol. 62, no. 7, pp. 4044 4055, July 2015.

[10] J. Richter, P. Bauerle, T. Gemassmer, and M. Doppelbauer, "Transient trajectory control of permanent magnet synchronous machines with nonlinear magnetics," in IEEE International Conference on Industrial Technology (ICIT), March 2015, pp. 2345-2351.

[11] A. Schmitt, M. Gommeringer, J. Kolb, and M. Braun, "A high current, high frequency modular multiphase multilevel converter for power hardware-in-the-loop emulation," in International Exhibition and Conference for Power Electronics, Intelligent Motion, Renewable Energy and Energy Management; Proceedings of PCIM Europe, 5 2014, pp. 1-8. 